\title{
Betriebsnahe Versorgungsnetzwerke und -angebote zur Unterstützung des Return to Work nach psychischer Krise: Zwischen gesundheitsförderlicher Organisationsentwicklung und Selbstmanagement der Zurückkehrenden
}

\author{
Workplace and Work-Related Return to Work Interventions for \\ Employees with Common Mental Disorders: Between Health- \\ Promoting Organisational Development and Self-Management
}

Autoren

Betje Schwarz, Ralf Stegmann, Uta Wegewitz

Institut

Bundesanstalt für Arbeitsschutz und Arbeitsmedizin Standort Berlin, Fachgruppe 3.3 „Evidenzbasierte Arbeitsmedizin, Betriebliches Gesundheitsmanagement“

\section{Schlüsselwörter}

betriebliche Wiedereingliederung, psychische Erkrankungen, qualitative Forschung

Key words

return to work, common mental disorders, qualitative research

Online publiziert: 1.9 .2020

Bibliografie

Rehabilitation 2020; 59: 263-272

DOI 10.1055/a-1194-0114

ISSN 0034-3536

(C) 2020. Thieme. All rights reserved.

Georg Thieme Verlag KG, Rüdigerstraße 14,

70469 Stuttgart, Germany

Korrespondenzadresse

Dr. Betje Schwarz

Deutsche Rentenversicherung Berlin-Brandenburg

Abteilung Rehabilitation und Gesundheitsförderung

Knobelsdorffstraße 92

14047 Berlin

betje.schwarz@drv-berlin-brandenburg.de

丹

Die Online-Tab. 2 und 3 finden Sie unter
https://doi.org/10.1055/a-1194-0114

\section{ZUSAMMENFASSUNG}

Ziel der Studie Noch immer bilden Kooperationen zwischen medizinisch-therapeutischen Einrichtungen und Betrieben sowie arbeitsplatzbezogene Interventionen für Beschäftigte mit psychischen Krisen und Erkrankungen Ausnahmen in der nationalen Versorgung. Die vorliegende Studie untersucht einige dieser Ausnahmen mit dem Ziel, ihre Vernetzungsformen und (Be-)Handlungsansätze zu beschreiben, ihre Stärken und Grenzen zu identifizieren und so wichtige Hinweise zu ihrer Weiterentwicklung und Dissemination zu geben.

Methodik Auf Basis einer Onlinerecherche wurden 5 Angebote ausgewählt. Anschließend wurden 11 Gruppendiskussionen und 17 Interviews mit den in diese Angebote involvierten betrieblichen und überbetrieblichen Akteuren $(n=44)$ sowie mit Betroffenen ( $n=27$ ) durchgeführt und mittels dokumentarischer Methode der Interpretation bzw. qualitativ-inhaltsanalytisch ausgewertet.

Ergebnisse Es ließen sich 2 (Be-)Handlungsansätze und Vernetzungsformen identifizieren. Ein individuumsbezogener Selbstmanagementansatz und ein systemischer Fallmanagementansatz. Im Fokus des ersten Ansatzes steht die Frage, was der Betroffene tun muss, um an seinen Arbeitsplatz zurückkehren zu können, der zweite Ansatz fragt zusätzlich danach, was hierfür am Arbeitsplatz getan werden kann. Geprägt werden die Ansätze u. a. durch die (be-)handlungsleitenden Orientierungsrahmen der beteiligten Akteure, wie z. B. deren Annahmen zur Veränderbarkeit arbeitsbezogener Risikofaktoren. Die größte Stärke des individuumsbezogenen Ansatzes liegt in der Stärkung der Selbstsorge, seine größte Begrenzung im einseitigen Fokus auf die individuelle (An-)Passung und der damit einhergehenden Gefahr der Individualisierung von Erkrankung, Ursachen und Bewältigung. Der systemische Ansatz verspricht Nachhaltigkeit durch die Kombination individuumsbezogener und betrieblicher Maßnahmen. Die Herausforderung hier besteht darin, die richtige Balance zwischen individueller Selbstund betrieblicher Fürsorge zu finden.

Schlussfolgerung Die Ergebnisse zeigen, wie psychisch erkrankte Beschäftigte mittels vernetzter und arbeitsplatzbezogener Angebote bei ihrer Wiedereingliederung unterstützt werden können. Sie zeigen jedoch auch, an welche Grenzen bisherige Ansätze stoßen und geben damit wichtige Hinweise für deren Weiterentwicklung und Dissemination. 


\section{ABSTRACT}

Purpose Work-related interventions and alliances between healthcare institutions and companies that aim to support employees with common mental disorders (CMD) returning to work are still quite rare in Germany. The present study examines a small sample of existing alliances and interventions with the aim to describe their cooperation forms and treatment approaches, to identify their strengths and weaknesses, and thus to provide guidance for their further development and dissemination.

Methods Five alliances/interventions were selected on the basis of a web search. Subsequently 11 group discussions and 17 qualitative interviews with involved health care professionals and occupational stakeholders $(n=44)$ as well as employees with CMD $(n=17)$ were conducted and evaluated by documentary method and qualitative content analysis.

Results The examined interventions follow either a person oriented self-management or a systemic case management approach. The self-management approach focusses on what has to be done by the person him-/herself to get back to work (focus on individual adaption). The systemic approach additionally asks for workplace adaption. Which approach is chosen, depends inter alia on the involved actors' preconscious frames of orientation, i. e. their assumptions regarding the modifiability of work-related risk factors. The main strength of the selfmanagement approach lies in its ability to reinforce self-care, its main shortcoming in the one-sided focus on individual adaption and the risk of individualizing the disease, its causes and coverage. The systemic approach seems to be more sustainable through the combination of individual and workplace adaptions. The challenge here is to find an appropriate balance between individual self-care and occupational health care.

Conclusion The findings show how the RTW of employees with CMD can be supported by more collaborative and work-related interventions. However, they also reveal limits and barriers of existing interventions and therefore provide useful hints for their further development and dissemination.

\section{Einleitung}

Psychische Krisen und Erkrankungen sind weit verbreitet und häufig Ursache vielfältiger Beeinträchtigungen, insbesondere auch im Bereich der Erwerbsarbeit [1]. So bilden sie die zweithäufigste Ursache von Arbeitsunfähigkeit, gehen mit doppelt so langen Fehlzeiten wie andere Erkrankungen einher und sind der häufigste Grund für Erwerbsminderungsrenten [2].

Ungeachtet der Belege, dass sich Belastungen am Arbeitsplatz negativ auf das Wohlbefinden und die psychische Gesundheit Beschäftigter auswirken können [3], ist Erwerbsarbeit aufgrund ihrer zentralen Bedeutung für Existenzsicherung, Zeitstrukturierung, soziale Einbindung und Anerkennung sowie persönliche Identität immer auch eine wichtige psychosoziale Ressource [4]. Fallen Menschen aufgrund einer psychischen Krise oder Erkrankung in längere Arbeitsunfähigkeit, Arbeitslosigkeit oder Erwerbsminderung, kann dies zur weiteren Verschlechterung ihres Gesundheitszustandes sowie ihrer gesellschaftlichen Teilhabe führen.

Psychische Erkrankungen sind mit ihren Auswirkungen auf die Arbeits- und Erwerbsfähigkeit und den damit verbundenen hohen Krankheitskosten auch in volks- wie betriebswirtschaftlicher Hinsicht von besonderer Relevanz [5]. Neben den Institutionen des Gesundheits- und Sozialversicherungssystems haben daher insbesondere auch Betriebe ein Interesse am Erhalt der psychischen Gesundheit ihrer Beschäftigten.

Um bestehende Defizite in der Regelversorgung (wie z. B. die verspätete Einleitung psychosomatischer Rehabilitation sowie die fehlende Abstimmung zwischen den an Behandlung und Wiedereingliederung beteiligten Akteuren) zu überwinden und betroffene Beschäftigte zeitnah und bedarfsorientiert versorgen zu können, wurden v. a. in der jüngeren Vergangenheit seitens einzelner Betriebe, psychosozialer Anlauf- und Beratungsstellen, medizinisch-therapeutischer Einrichtungen und teilweise auch Leistungsträger (Krankenkassen, Rentenversicherungsträger) spezielle, zumeist lokal oder regional verortete, Versorgungsnetzwerke und/ oder Beratungsangebote mit besonderem Arbeitsplatzbezug entwickelt [6].

Aus internationalen Studien liegen erste Befunde vor, dass solche stärker vernetzten und arbeitsplatzbezogenen Angebote für Menschen mit psychischen Krisen und Erkrankungen nicht nur im Hinblick auf gesundheits-, sondern auch bezüglich arbeitsbezogener Zielgrößen besonders vielversprechend sind. So beschleunigen sie den Prozess des Return to Work (RTW) und sichern nachhaltig die berufliche Teilhabe [7-11].

Trotz dieser Befunde bleibt die Umsetzung in Deutschland bisher auf wenige Modellprojekte begrenzt. Aufgrund fehlender wissenschaftlicher Untersuchungen liegen zu den bestehenden Netzwerken und Angeboten zudem nur wenig empirisch gewonnene Erkenntnisse vor $[6,12,13]$.

Die vorliegende von der Hans-Böckler-Stiftung im Rahmen des Forschungsverbundes „Neue Allianzen zum Erhalt der Beschäftigungsfähigkeit“ (Projekt-Nr.: 2016-980-4) geförderte Studie „Rückkehr zur Arbeit nach einer psychischen Krise - Vernetzung von betrieblichen und klinischen Akteuren im Return to Work-Prozess“ der Bundesanstalt für Arbeitsschutz und Arbeitsmedizin (BAuA) setzt hier an und nimmt eine Auswahl der in den letzten Jahren bundesweit modellhaft entstandenen Versorgungsnetzwerke und -angebote aus Sicht Betroffener sowie aus der Perspektive der an ihnen beteiligten (über-)betrieblichen Akteure vertiefend in den Blick [14].

Die Identifikation von Angeboten erfolgte auf Basis einer Internetrecherche und die Auswahl folgte dem Anliegen, 4 bis 5 in ihrer Art und ihren Inhalten möglichst heterogene und kontrastive Angebote für die Studie zu berücksichtigen. Die beteiligten überbetrieblichen Einrichtungen sollten zudem unterschiedlichen Versorgungszweigen und -formen angehören (Anlauf- und Beratungsstellen, akutpsychiatrische und psychotherapeutische Einrichtungen, Reha-Einrichtungen, ambulante, teilstationäre und stationäre Einrichtungen) und die beteiligten Betriebe in Branche und Größe va- 
riieren. Die Anzahl von 4 bis 5 Angeboten wurde unter dem Gesichtspunkt der Machbarkeit (zu bewältigende Datenmenge für die zeitlich begrenzte qualitative Studie) sowie der Annahme gewählt, dass damit zugleich die angestrebte Varianz zwischen den Angeboten und mit dieser ein guter Grad der theoretischen Sättigung von Daten und Ergebnissen erreicht werden kann.

Ziel war es, die ausgewählten Versorgungsangebote mit ihren jeweiligen Vernetzungsformen und (Be-)Handlungsansätzen zu beschreiben, Gelingensbedingungen sowie Stärken und Grenzen zu identifizieren sowie auf dieser Basis Hinweise zur (Weiter-)Entwicklung solcher RTW-Strategien abzuleiten.

\section{Material und Methoden}

Um die ausgewählten Versorgungsangebote im Hinblick auf die oben genannte Zielstellung vertiefend untersuchen zu können, wurde ein qualitatives Studiendesign gewählt, mit dem sowohl die expliziten (d. h. reflexiv-verfügbaren) als auch die impliziten (d. h. vorbewusst-atheoretischen) Erfahrungs- und Wissensbestände der involvierten (über-)betrieblichen Akteure sowie Betroffenen erhoben und rekonstruiert werden konnten.

Während explizite Erfahrungs- und Wissensbestände direkt erfragt bzw. unmittelbar aus manifesten Gesprächsinhalten (dem ,WAS' gesagt wird) abgeleitet werden können, müssen implizite Erfahrungs- und Wissensbestände mittels selbstläufiger (d.h. mit nur wenig äußerem Zutun des Forschenden sich entwickelnde) Narrationen oder Diskurse erhoben und anschließend systematisch aus dem ,WIE' des Erzählten rekonstruiert werden [15-18]. Zur Erhebung beider Erfahrungs- und Wissensbestände wurden Gruppendiskussionen und Telefoninterviews eingesetzt, die durch erzählgenerierende Fragen eröffnet, durch immanente (an bereits Gesagtes anknüpfende) Nachfragen am Laufen gehalten sowie durch exmanente (vom Erkenntnisinteresse geleitete) Zusatzfragen ergänzt wurden. Als Auswertungsmethoden kamen die dokumentarische Methode der Interpretation [15-18] sowie ergänzend ein qualitativ-inhaltsanalytisches Verfahren $[19,20]$ zum Einsatz. Grundlage aller Analysen bildeten die pseudonymisierten Transkripte der von den Gruppendiskussionen und Telefoninterviews angefertigten Audioaufzeichnungen.

\section{Realisiertes Sample}

- Tab. 1 gibt einen Überblick über die für die Studie ausgewählten Angebote. Wie der Tabelle entnommen werden kann, konnte die zur Maximierung der Varianz, Kontrastivität und theoretischen Sättigung angestrebte Heterogenität sowohl im Hinblick auf die Art und die Inhalte der Angebote als auch in Bezug auf die beteiligten überbetrieblichen Einrichtungen erreicht werden. So finden sich unter den Angeboten psychosomatische Sprechstunden, berufsorientierte Therapieangebote, Reha-Netzwerke, Beratung zum Return to Work (RTW) und Betrieblichen Eingliederungsmanagement (BEM) sowie Berufscoachingansätze, die von psychosozialen Anlauf- und Beratungsstellen, psychotherapeutischen und psychiatrischen Ambulanzen und Kliniken sowie von Reha-Zentren vorgehalten und durchgeführt werden. Auf Seite der Betriebe konnten zwar Unternehmen unterschiedlicher Branchen für die Studie gewonnen werden, jedoch ist es nicht gelungen, neben großen und einem mittelständischen auch kleine Unternehmen (die von 2 der untersuchten Angebote durchaus Gebrauch machen bzw. mit denen Kooperationen zu den aufgeführten medizinisch-therapeutischen Einrichtungen bestehen) zur aktiven Teilnahme an der Studie zu bewegen.

Nach Auswahl der Angebote erfolgte die Terminierung der Gruppendiskussionen und Telefoninterviews, die Festlegung der konkreten Teilnehmenden auf Betriebs- und Einrichtungsseite (Vorgabe war, dass möglichst alle für das jeweilige Angebot relevanten betrieblichen Akteure bzw. Professionen teilnehmen; die Ansprache und Benennung der Teilnehmenden oblag dann aber den Betrieben bzw. Einrichtungen selbst) sowie die Information und Rekrutierung der Betroffenen (hier erfolgte die Ansprache über die Einrichtungsseite bzw. in Einzelfällen auch über die Betriebsärzte). Der Einschluss in die Studie erfolgte ausschließlich nach ausführlicher schriftlicher und mündlicher Information sowie bei Vorliegen der schriftlichen Einwilligungserklärung.

Im Zeitraum von Juni 2017 bis Mai 2018 wurden 11 Gruppendiskussionen und 17 Telefoninterviews mit insgesamt 44 betrieblichen und überbetrieblichen Akteuren sowie 27 Betroffenen durchgeführt (vgl. > Tab. 2 sowie > Tab. $\mathbf{3}$ (beide online)). Die Auswertungen erfolgten im Zeitraum von Oktober 2017 bis Dezember 2018 - bei den Gruppendiskussionen mittels dokumentarischer Methode [15-18], bei den Telefoninterviews qualitativ inhaltsanalytisch [19, 20]. Den Analysen lagen 969 Transkriptseiten von 2148 min Gesprächsaufzeichnungen zugrunde.

\section{Datenschutz und Ethik}

Zu Beginn der Studie wurden ein ausführliches Datenschutzkonzept sowie ein Ethikantrag erstellt. Ersteres wurde durch die Datenschutzbeauftragte der BAuA, letzterer durch die Ethikkommission der Medizinischen Hochschule Hannover geprüft und positiv beschieden (Votum-Nr. 7490). Die Datenerhebung erfolgte erst nach Eingang der positiven Voten.

\section{Ergebnisse}

\section{Vernetzungsformen und (Be-)Handlungsansätze}

In der Analyse zeigte sich, dass die Vernetzung zwischen betrieblichem und medizinisch-therapeutischem System im konkreten Einzelfall bei keinem der Angebote direkt, also über den unmittelbaren Kontakt beider Akteursgruppen, sondern immer indirekt, entweder vermittelt über den Betroffenen oder über einen externen Dritten (beratend tätigen, vom Arbeitgeber oder einem Leistungsträger beauftragten, Akteur), erfolgt ( $\triangleright \mathbf{A b b} \mathbf{1} \mathbf{1})$.

Den beiden Vernetzungsformen (indirekte Vernetzung über den Betroffenen vs. indirekte Vernetzung über einen externen Dritten) ließen sich zudem 2 unterschiedliche (Be-)Handlungsansätze zuordnen: ein psychotherapeutisch orientierter individuumsbezogener Selbstmanagementansatz (indirekte Vernetzung über den Betroffenen) und ein sozialarbeiterisch orientierter systemischer Fallmanagementansatz (indirekte Vernetzung über den externen Dritten).

Während der Arbeitsplatzbezug im individuumsbezogenen Selbstmanagementansatz im Wesentlichen dadurch hergestellt wird, dass die Arbeit als Thema und die betriebliche Wiedereingliederung als Ziel in die Intervention hinein geholt werden, findet die 
- Tab. 1 Untersuchte Versorgungsnetzwerke und -angebote (vgl. [14]).

\begin{tabular}{|c|c|c|c|}
\hline Zif. & Beteiligte & Angebot & Kurzbeschreibung \\
\hline 1 & $\begin{array}{l}\text { Großunternehmen (Stahlindustrie), } \\
\text { Einrichtung der psychotherapeutischen } \\
\text { Versorgung, Krankenkasse }\end{array}$ & $\begin{array}{l}\text { Psychosomatische } \\
\text { Sprechstunde }\end{array}$ & $\begin{array}{l}\text { Frühzeitige Identifikation und Zuweisung betroffener Mitarbeiter } \\
\text { durch Betriebsärzte/Fallmanager der Krankenkasse; Sprechstunde: } \\
\text { psychologische Diagnostik, Krisenintervention, Vermittlung in die } \\
\text { Regelversorgung (Psychotherapie, akutpsychiatrische Behandlung, } \\
\text { psychosomatische Reha) }\end{array}$ \\
\hline 2 & $\begin{array}{l}\text { Großunternehmen (Logistik), Reha- } \\
\text { Einrichtung, Krankenkasse }\end{array}$ & $\begin{array}{l}\text { Betriebsärztlich eingeleitete } \\
\text { medizinisch-beruflich } \\
\text { orientierte psychosomatische } \\
\text { Reha (MBO-PS-Reha) }\end{array}$ & $\begin{array}{l}\text { Frühzeitige Identifikation betroffener Mitarbeiter durch Betriebsärz- } \\
\text { te, Sozialberatung, BEM-Beauftragte; Einleitung der MBO-PS-Reha } \\
\text { durch Betriebsärzte (gutachterliche Stellungnahme, Terminierung); } \\
\text { Betriebsärzte erhalten kurzen Entlassungsbericht per Fax als } \\
\text { Grundlage für das BEM }\end{array}$ \\
\hline 3 & $\begin{array}{l}\text { Mittelständische u. Großunternehmen } \\
\text { versch. Branchen (davon an der Studie } \\
\text { beteiligt: Großunternehmen der } \\
\text { Konsumgüterproduktion), Einrichtung } \\
\text { der psychiatrischen, psychotherapeuti- } \\
\text { schen und psychosomatischen } \\
\text { Versorgung, versch. Krankenkassen }\end{array}$ & $\begin{array}{l}\text { Psychosomatische Sprechstun- } \\
\text { de, berufsorientierte } \\
\text { ambulante, teil-stationäre u. } \\
\text { stationäre Therapie-angebote, } \\
\text { Return to Work (RTW)- Bera- } \\
\text { tung nach akutpsychiatrischer } \\
\text { Behandlung }\end{array}$ & $\begin{array}{l}\text { Frühzeitige Identifikation und Zuweisung betroffener Mitarbeiter } \\
\text { durch Betriebsärzte, Sozialberatung, Human Resources, BEM-Beauf- } \\
\text { tragte; Sprechstunde: psychologische Diagnostik, Kriseninterventi- } \\
\text { on, Vermittlung in die Regelversorgung (Psychotherapie, akutpsych- } \\
\text { iatrische Behandlung, psychosomatische Reha); RTW-Beratung für } \\
\text { arbeitsunfähig aus der akutpsychiatrischen Behandlung entlassene } \\
\text { Patienten }\end{array}$ \\
\hline 4 & $\begin{array}{l}\text { Kleinst-, Klein- u. mittelständische } \\
\text { Unternehmen versch. Branchen (keine } \\
\text { Beteiligung an der Studie), Einrichtung } \\
\text { für Prävention u. Rehabilitation, } \\
\text { regionaler Rentenversicherungsträger }\end{array}$ & $\begin{array}{l}\text { Berufscoaching nach } \\
\text { medizinisch-beruflich } \\
\text { orientierter psychosoma-tisch- } \\
\text { er Reha (MBO-PS-Reha) }\end{array}$ & $\begin{array}{l}\text { Berufscoaching nach einer MBO-PS-Reha für Patienten mit weiterge- } \\
\text { hendem Unterstützungsbedarf im Hinblick auf die Wiedereingliede- } \\
\text { rung }\end{array}$ \\
\hline 5 & $\begin{array}{l}\text { Regional ansässige Unternehmen versch. } \\
\text { Größen u. Branchen (davon an der } \\
\text { Studie beteiligt: ein Großunternehmen } \\
\text { der Pharmaindustrie und ein mittelstän- } \\
\text { discher Interessensverband aus dem } \\
\text { Gesundheitswesen), } 3 \text { Psychosoziale } \\
\text { Anlauf-/Beratungs-stellen, versch. } \\
\text { Sozialversicherungsträger }\end{array}$ & $\begin{array}{l}\text { Psychosoziale Beratung, } \\
\text { Krisen-intervention, Vermitt- } \\
\text { lung in die Regelversorgung, } \\
\text { Berufs- und BEM-Beratung u. } \\
\text { Begleitung }\end{array}$ & $\begin{array}{l}\text { Psychosoziale Beratung, Krisenintervention, Vermittlung in } \\
\text { Regelversorgung (Psychotherapie, akutpsychiatrische Behandlung, } \\
\text { psychosomatische Reha), Berufs- und BEM-Beratung und Begleitung } \\
\text { [Bei einer der Anlauf- und Beratungsstellen ist das Angebot der } \\
\text { Berufs-/BEM-Beratung und Begleitung bei Beschäftigten mit } \\
\text { Schwerbehinderung oder Gleichstellung bereits Teil der Regelver- } \\
\text { sorgung. Weitere Personengruppen partizipieren bislang nur im } \\
\text { Falle einer arbeitgeberseitigen Finanzierung]. }\end{array}$ \\
\hline
\end{tabular}

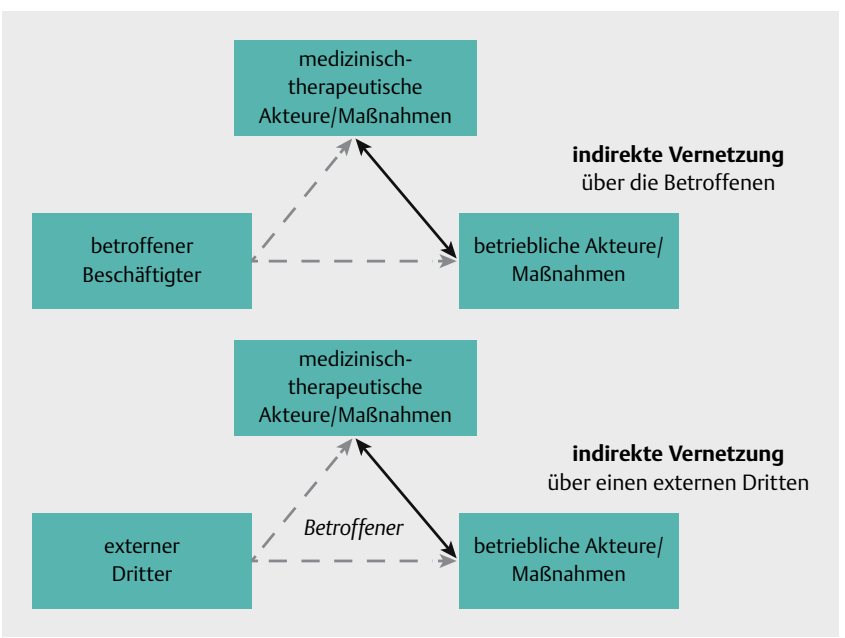

- Abb. 1 Vernetzungsformen.

Intervention im systemischen Fallmanagementansatz unmittelbar, also direkt am Arbeitsplatz unter Einbezug relevanter betrieblicher Akteure statt. Entsprechend liegt der Fokus des ersten Ansatzes auf der individuellen, der des zweiten zusätzlich auf der betrieblichen (An-)Passung. Dies spiegelt sich auch in den (be-)handlungsleitenden Fragen der beiden Ansätze wider. So fragt der individuumsbezogene Ansatz danach, was der Betroffene tun muss, um an seinen Arbeitsplatz zurückkehren zu können (Änderung individueller Haltungen, Einstellungen und Verhaltensweisen; Bearbeitung privater Probleme; Stärkung von Selbstsorge und Selbstmanagementkompetenz), der systemische Ansatz zusätzlich, was hierfür am Arbeitsplatz getan werden kann (Änderung von Arbeitsanforderungen und -bedingungen; Klärung von Konflikten/Gestaltung von Arbeitsbeziehungen).

Die folgenden Zitate veranschaulichen die Spezifika und Unterschiede beider Ansätze.

Zitate von (über-)betrieblichen Akteuren aus Angeboten mit individuumsbezogenem Selbstmanagementansatz:

„Also ich bin immer etwas berüchtigt mit der Frage: ,Wie können Sie sich verändern, dass Sie unter der gegebenen Organisation weniger leiden? [...] Was kann ich dafür tun, mit meinem Verhalten, mit meinen Strategien, dass das zwischen mir und meinem Arbeitsplatz später gesund harmoniert? Und da hab ja ich die beste Kontrolle über mein eigenes Verhalten ““. 
„[Es geht darum] die Selbstfürsorge, Abgrenzungsfähigkeit zu stärken; da muss was im Kopf der Menschen passieren, die die Krankheit haben“.

„[...] dass systematisch der Arbeitsplatz fokussiert wird, welche Ziele bezogen auf Arbeit sollen verfolgt werden und welche Fertigkeiten und Fähigkeiten muss die Person haben, um am Arbeitsplatz zu bleiben oder wiedereingegliedert zu werden. Und Interventionen am Arbeitsplatz werden mittelbar durchgeführt, in Form von kleineren Rollenspielen oder Hilfestellungen, der Therapeut den Patienten entsprechend instruiert, indem neue Gedanken eingeübt werden und der Patient durch gecoacht wird“.

Zitate von (über-)betrieblichen Akteuren aus Angeboten mit systemischem Fallmanagementansatz:

„[... ] also ist für mich immer die generelle Frage: was braucht [die betroffene Person] um gut arbeiten zu können? [...] Wir versuchen Lösungen zu finden und müssen natürlich auch sehen, was ist im Betrieb möglich. [...] Da muss man sich erst mal Stück für Stück an die Vorgesetzten und BEM-Beauftragten ranrobben: ,Wir bieten was an, das ist nicht destruktiv, sondern hilft Lösungen zu finden'. [...] Und dieses Begleiten in die Betriebe [...], das ist was der Facharzt oder auch der Psychotherapeut häufig überhaupt nicht leisten können“.

„[...] dass man so die Funktion des Dritten hat, der dazu kommt und die Dinge erst mal auch transparent macht [...] die Konfliktpunkte und die Möglichkeiten [...] Und da ist unsere Erfahrung, dass sich solche Knotenpunkte dann auch lösen lassen, weil plötzlich hingeschaut werden kann“.

\section{Einflussfaktoren auf Vernetzungsformen und (Be-) Handlungsansätze}

Die Analyse zeigte ferner, dass die beiden identifizierten Ansätze durch bestimmte Einflussfaktoren in ihrer jeweiligen Charakteristik geprägt werden. Neben Faktoren, die den (Be-)Handlungsauftrag (das ,Sollen und Dürfen'), die (Be-) Handlungsbereitschaft (das ,Wollen') und die (Be-)Handlungsmacht (das ,Können') der (über-) betrieblichen Akteure bestimmen (wie z. B. der (leistungs-)rechtliche Rahmen und daraus erwachsene Zuständigkeiten und Verantwortlichkeiten, eigene Interessen und Ziele sowie vorhandene Kompetenzen und Ressourcen), wirken sich insbesondere die (be-) handlungsleitenden Orientierungsrahmen beider Akteursgruppen auf Vernetzungsform und (Be-)Handlungsansatz aus. Von besonderer Relevanz zeigten sich folgende drei Orientierungsrahmen ( $>$ Tab. 4): 1. Krankheitstheorien (Bedeutung arbeits- und personenbezogener Risikofaktoren sowie privater Belastungen), 2. Annahmen über die Veränderbarkeit arbeitsbezogener Risikofaktoren und 3. Annahmen mit Blick auf Betroffene (Betroffenenbilder).

\section{Krankheitstheorien, Orientierungsrahmen und Handlungsstrategien der Betroffenen}

Wie in $>$ Tab. 5 dargestellt, beschreiben die Betroffenen ihre Erkrankungen als Folge der Wechselwirkung und Aufschichtung vielfältiger Faktoren: personenbezogener Risikofaktoren (z. B. hohes Pflicht- und Verantwortungsbewusstsein, starker Perfektionismus), privater Probleme und biographischer Ereignisse (z. B. schlechten Kindheitserfahrungen oder Trennungen) sowie arbeitsbezogener Belastungen und Konflikte (z. B. hoher Arbeitsmenge, Konflikte mit Vorgesetzten, Mobbing durch Kollegen).
Aus den Erzählungen der Betroffenen wird deutlich, dass die verschiedenen Faktoren - je nach (Be-)Handlungsansatz - unterschiedlich bearbeitet werden. So erzählen Betroffene aus individuumsbezogenen Ansätzen v. a. von der Reflexion personenbezogener Risikofaktoren, privater Belastungen und biografischer Ereignisse sowie dem Erlernen entsprechender Bewältigungsstrategien. Betroffene, die einen systemischen Ansatz erlebt haben, berichten ergänzend dazu auch von der gezielten Identifikation arbeitsbezogener Risikofaktoren und deren professionell begleiteter Anpassung.

Die unterschiedliche Bearbeitung der Risikofaktoren in den beiden (Be-)Handlungsansätzen scheint dazu zu führen, dass die Betroffenen im Rahmen ihrer Therapie die dort jeweils vorherrschenden Orientierungsrahmen übernehmen und entsprechende Handlungsstrategien für sich ableiten. So gewinnen bei Betroffenen aus individuumsbezogenen (Be-)Handlungsansätzen personenbezogene Faktoren sowie private und biografische Belastungen in ihren Krankheitstheorien an Bedeutung, werden arbeitsbezogene Risikofaktoren stärker als unveränderbar wahrgenommen und die erfolgreiche Rückkehr an den Arbeitsplatz v. a. an die eigene Person und die individuellen Anpassungsbemühungen gebunden: „Ich bin ja derjenige, der es bestimmt. Wenn ich mich nicht verändere, kann mir auch keiner helfen“.

Bei Betroffenen aus systemischen (Be-)Handlungsansätzen bleiben die Krankheitstheorien komplexer - private, biografische, personen- und arbeitsbezogene Risikofaktoren gelten als gleichermaßen bedeutsam. Die Wiedereingliederung kann aus ihrer Perspektive daher nur gelingen, wenn alle Faktoren bearbeitet werden und ein neues Gleichgewicht über individuelle und betriebliche (An-) Passung hergestellt werden kann: „Ohne die Bereitschaft der Beteiligten, das zu einem guten Verlauf und Ende zu bringen, kann so was gar nicht funktionieren. [...] Also es ist so ein Gesamtkonzept für mich gewesen. Ein privates Umfeld, was das mitträgt, dann die Frau A. [vom Integrationsfachdienst], die Schwerbehindertenbeauftragte, und eben auch der Arbeitgeber, der versucht hat: ,Was machen wir jetzt damit? ‘ [...] Und dann eben auch: ,Suchen Sie sich eine Psychotherapeutin - okay, hab ich; und auch noch eine Reha - ja, gut.' [...] In der Zeit, wo ich in der Reha war, hat der Personalchef geguckt, wo sie mich jetzt am besten unterbringen. [...] wo der Arbeitgeber auch Verantwortung wahrgenommen hat, das fand ich toll. Also das ist ein Puzzle von allem gewesen“.

\section{Diskussion}

\section{Stärken und Grenzen der Vernetzungsformen und (Be-)Handlungsansätze}

Den untersuchten Angeboten ist gemein, dass sie durch die Vernetzung des betrieblichen und medizinisch-therapeutischen Systems sowie eines verstärkten Arbeitsbezugs ihrer Interventionen bestehende Defizite in der Regelversorgung psychisch erkrankter Beschäftigter überwinden, Betroffene häufig frühzeitiger erreichen und sie gezielter auf die betriebliche Wiedereingliederung vorbereiten können.

Die beiden identifizierten Vernetzungsformen und (Be-)Handlungsansätze besitzen dabei ganz spezifische Stärken, weisen aber auch Begrenzungen auf. 
- Tab. 4 (Be-)Handlungsleitende Orientierungsrahmen betrieblicher und überbetrieblicher Akteure (vgl. [14]).

\begin{tabular}{|c|c|}
\hline & Individuumsbezogener Selbstmanagementansatz \\
\hline $\begin{array}{l}\text { Krankheits- } \\
\text { theorien }\end{array}$ & $\begin{array}{l}\text { - Personenbezogene Risikofaktoren (insb. ungünstige arbeitsbezogene Erlebens- } \\
\text { und Verhaltensweisen) sowie private Belastungen und Konflikte als primäre } \\
\text { Ursachen psychischer Krisen und Erkrankungen } \\
\text { " Arbeitsbezogene Risikofaktoren und Probleme als hinzukommende (sekundäre, } \\
\text { allein nicht hinreichende) Belastungsfaktoren: ,Arbeit an sich macht nicht krank } \\
\text { Zitate: } \\
\text { „[...] oft ist es gar nicht die Arbeit an sich, da regt man sich zwar auf oder das nimmt } \\
\text { man auch gerne als Ausrede, aber im privaten Bereich, da sind dann schon ganz viele } \\
\text { Dinge erkennbar“. } \\
\text { „Der Veränderungsprozess [im Unternehmen] ist unheimlich schwer für die } \\
\text { [langjährigen älteren Mitarbeiter] zu akzeptieren. Das ist glaub ich der größte Faktor, } \\
\text { der momentan an der Basis da uns die Erkrankung schafft. Und vieles wird dann, was } \\
\text { zu Hause auch noch passiert, mit reingebracht, und natürlich der erste Ableiter für } \\
\text { so was ist nämlich dann der Arbeitgeber, ne? Der ist dann dafür verantwortlich, aber } \\
\text { eigentlich der Ursprung oder der Keim ist ganz woanders gelegt worden“. }\end{array}$ \\
\hline
\end{tabular}

\begin{tabular}{l|l}
$\begin{array}{l}\text { (Un-) } \\
\text { Veränderbar- }\end{array}$ & - Arbeitsbedingungen als unveränderbare Strukturen \\
$\begin{array}{l}\text { keit arbeits- } \\
\text { bezogener }\end{array}$ & - Veränderungen nur langfristig durch Politik und Wirtschaft möglich \\
$\begin{array}{l}\text { Risikofaktoren } \\
\text { - Medizinisch-therapeutische Akteure können hier nichts tun, haben diesbezüglich }\end{array}$ & keine Handlungsmacht
\end{tabular}
Zitate:

„Man kann ja auch keine Arbeitswelt, Strukturen schaffen, die die es hier nicht gibt. An bestimmte Arbeitsplätze sind bestimmte Anforderungen verbunden, und die sind jetzt nicht alle weg, ne?“

„Wir haben nicht ein Job, wo man sagen kann jetzt brauchst du nur achtzig Prozent hier leisten. ja? [...] Also als [Berufsgruppe] ist man hundert Prozent einsatzfähig oder nicht einsatzfähig. es gibt kein bisschen einsatzfähig“.

„Wir müssen mit diesen schwierigen Rahmenbedingungen ja auch arbeiten [...], die sind Teil dieser ganzen gesellschaftlichen Position. Also wir sind nicht diejenigen, ne? Auch wir sehen natürlich die Schwierigkeiten und würden uns auch wünschen, dass das zurückgemeldet wird an entsprechende Stellen“.

„Die Bedingungen können wir nicht therapieren. [...] Politisch muss man das angehen; aber das sollte man nicht über den Patienten tun [...] das wär eine Überforderung für den Patienten, also der braucht ja jetzt in der Situation Hilfe“.

Bilder von Betroffenen
- Betroffene als ,Brenner/Ausbrenner': geraten aufgrund ihrer zu geringen Abgrenzungs- und Distanzierungsfähigkeit, zu hohen Verausgabungsbereitschaft, ihres überhöhten Perfektionsstrebens und ihrer mangelnden Selbstfürsorge in eine zunehmende Belastungs- und Erschöpfungssituation, die schließlich in ein Burn Out bzw. eine Erschöpfungsdepression mündet Zitat:

„Es werden immer mehr jüngere Mitarbeiter krank. Die legen sich selber Arbeitspensen auf durch eigenen Ehrgeiz, Perfektionismus, oder Erwartungen [...], wo auch der Mitarbeiter gefragt ist. Wir reden immer über die Führungskraft. Also jeder Mensch hat auch eine Mitwirkpflicht “.

- Betroffene als,Gekränkte/Verbitterte‘: geraten aufgrund eines subjektiv erlebten Defizits an Anerkennung und Wertschätzung seitens Kollegen, Vorgesetzten und/ oder des Arbeitsgebers und einer daraus resultierenden Kränkung in eine Verbitterungsstörung; sie externalisieren die Ursachen ihrer Erkrankung, weisen ihren Kollegen, Vorgesetzten und/oder Arbeitsgebern die Schuld/Verantwortung für ihre Erkrankung zu und erwarten Wiedergutmachung; kann in einen Rentenwunsch münden (,Betroffene als Rentenbegehrer')

Zitate:

„In den Gesprächen stell ich schon oft fest; es gibt irgendwann mal eine tiefe Kränkung; keine Anerkennung mehr für die Leistung [...]. Dann kommt reaktiv ein Verbitterungssyndrom [...]. [Das sind dann die,] die den Betriebsfrieden mit diesem [...] ,Der hat mich krank gemacht. ', dieser Unerbittlichkeit ,Das steht mir zu.' zerstören [...], die Arbeit häufig als ausschließlich ursächlich für ihre Erkrankung sehen und sagen ,Dorthin kann ich nicht zurück; ich brauch nur noch Rente', ne?“, „Wir müssen da auch hinsehen, dass ein nicht unerheblicher Anteil mit dem festen Vorsatz kommt einer Freistellung [...]. Wo Geld verteilt wird, gibt es immer ein Begehren, was nicht immer gerechtfertigt ist. Und wenn das so ist; liegt ein so manifester Zielkonflikt vor; dass Psychotherapie eigentlich gar keine Indikation ist, streng genommen“.

\section{Systemischer Fallmanagementansatz}

- Arbeitsbezogene Risikofaktoren (,betriebliche Schieflagen') als eigenständige Ursachen psychischer Krisen und Erkrankungen Zitate:

„Und das ist so klar, dass diese Arbeitsintensität ein Faktor ist, ein Risikofaktor für Depressionen. Und das muss man dann auch benennen, das müssen wir ändern“.

„[...] egal, ob diejenige gesund ist oder nicht, also es ist ein grundsätzliches Problem der mangelnden Wertschätzung“.

„Wir haben immer mehr Fälle von psychischen Erkrankungen und es geht nicht darum den Arbeitnehmer fit zu machen. Klar müssen wir Kompetenzen schulen, aber wir müssen uns überlegen: ,Ist das noch menschliche Arbeit? “ Und da ist der Knackpunkt, wir haben eine Grenze erreicht, wir funktionieren als Menschen nicht so, nicht mit dieser Arbeitswelt. Die Passung stimmt nicht mehr; der Mensch ist begrenzt, er muss ein anderes Arbeitsumfeld haben“.

- Arbeitsbedingungen als (prinzipiell) veränderbare betriebsspezifische und individuelle Anforderungen

- Veränderungen sind möglich, setzen aber die Bereitschaft aller Beteiligten und ggf. Unterstützung bei der Umsetzung voraus

- Überbetriebliche Akteure können hier motivieren, informieren und beratend zur Seite stehen Zitat:

"Ja es gibt die äußeren Schranken, an denen wir erst mal nichts rütteln können, aber innerhalb gibt es dann doch nochmal, gerade wenn ein geschulter Fachmann dabei sitzt, der eine gewisse Kreativität, gewisses Wissen und einen objektiven Blick mitbringt, dann doch nochmal helfen kann, die Stellschrauben zu drehen".

- Betroffene als ,Seismographen betrieblicher Schieflagen': nehmen betriebliche Schieflagen aufgrund einer besonderen ,Sensibilität‘ eher wahr, erkranken aber auch eher an ihnen (,besondere Vulnerabilität'); liefern wichtige Hinweise über bestehende psychische Belastungen und Beanspruchungen im Betrieb, die für die präventive gesundheitsförderliche und menschgerechte Gestaltung der Arbeit gezielt genutzt werden könnten

Zitate:

„Menschen mit einer höheren psychischen Sensibilität haben ja auch einne besondere Rolle und Funktion in diesen Strukturen. Die sind diejenigen; die am ehesten auch was wahrnehmen [...]. Sie sind eigentlich so Seismographen; die kippen als erstes um. [...] weil sie sich Gedanken machen; andere sind stärker in der Lage sich abzugrenzen. Die lassen sich nicht so sehr davon berühren“.

„Ja, ja das ist ganz spannend, weil es ist ja oft so eine Denkweise, der Einzelne hat das Problem, aber er steht nur als Symbolfigur eigentlich“. 
- Tab. 5 Krankheitsursachen aus Betroffenensicht.

\begin{tabular}{|c|c|c|}
\hline personenbezogenen Risikofaktoren & $\begin{array}{l}\text { privaten Problemen und } \\
\text { biografischen Risikofaktoren }\end{array}$ & arbeitsbezogenen Risikofaktoren \\
\hline $\begin{array}{l}\text { " Hohes Pflicht- und/oder Verantwortungs- } \\
\text { bewusstsein; hohe Leistungsorientierung } \\
\text { " Hohe Identifikation mit dem Job; hohe } \\
\text { Verausgabungsbereitschaft } \\
\text { - Geringe Abgrenzungs-/Distanzierungsfähigkeit } \\
\text { (nicht abschalten können, nicht nein sagen } \\
\text { können) } \\
\text { - Perfektionismus; hoher Ehrgeiz } \\
\text { " Sich beweisen wollen } \\
\text { " Angewiesenheit auf externe Bestätigung } \\
\text { - Angst vor Zurückweisung, von allen gemocht, es } \\
\text { allen recht machen wollen... } \\
\text { - Durchhalteverhalten } \\
\text { - Versagensängste, keine Schwäche zeigen wollen }\end{array}$ & $\begin{array}{l}\text { - Trennung/Scheidung } \\
\text { - Schulden } \\
\text { - Konflikte in der Familie } \\
\text { - Erkrankungen/Todesfälle in } \\
\text { der Familie } \\
\text { - Schlechte/traumatisierende } \\
\text { Erfahrungen in Kindheit/ } \\
\text { Jugend (Vernachlässigung, } \\
\text { Gewalt, Sucht, Missbrauch) }\end{array}$ & $\begin{array}{l}\text { " Hohe Arbeitsintensität/-dichte, hohes Arbeitsvolumen } \\
\text { " Überschreitung von Arbeitszeiten, Überstunden, } \\
\text { keine geregelten Pausen, Entgrenzung (Erreich-/ } \\
\text { Verfügbarkeit auch zu Hause/am Wochenende) } \\
\text { - Geringer Personalschlüssel } \\
\text { " Zeit-/Leistungs-/Erfolgsdruck } \\
\text { - Hohe Verantwortung bei geringer Qualifikation } \\
\text { - Geringer Handlungsspielraum bei hohem Kontrollgrad } \\
\text { - Arbeitsplatzunsicherheit } \\
\text { - Geringe/keine Anerkennung und Wertschätzung } \\
\text { - Abwertung, Schikanen, Mobbing } \\
\text { " Konflikte mit Kollegen und/oder Vorgesetzten } \\
\text { - Führungsstil }\end{array}$ \\
\hline
\end{tabular}

> Tab. 6 Stärken und Grenzen der Vernetzungsformen und (Be-)Handlungsansätze.

\begin{tabular}{|c|c|c|}
\hline & $\begin{array}{l}\text { Indirekte Vernetzung über die Betroffenen Individu- } \\
\text { umsbezogener Selbstmanagementansatz }\end{array}$ & $\begin{array}{l}\text { Indirekte Vernetzung über einen externen Dritten Systemischer } \\
\text { Fallmanagementansatz }\end{array}$ \\
\hline Stärken & $\begin{array}{l}\text { - Expliziter Arbeitsbezug der medizinisch-therapeuti- } \\
\text { schen Intervention } \\
\text { - Betroffener bleibt Herr/Frau des Verfahrens (Stärkung } \\
\text { Selbstsorge und im Erfolgsfall der Selbstwirksamkeit); } \\
\text { Unabhängigkeit von Rahmenbedingungen }\end{array}$ & $\begin{array}{l}\text { - Koordination und Begleitung des gesamten RTW-Prozesses } \\
\text { " Ansprechpartner für Betroffene und Akteure beider Systeme; Modell insb. } \\
\text { auch für KMU } \\
\text { - Haltung: ,Parteiische Neutralität‘/'Allseitigkeit‘; Beobachterposition (sieht } \\
\text { blinde Flecken) } \\
\text { - Kombination individuums- und arbeitsplatzbezogener Maßnahmen/ } \\
\text { beidseitige (An-)Passung } \\
\text { - Nachhaltigkeit (individuell/betrieblich) }\end{array}$ \\
\hline Grenzen & $\begin{array}{l}\text { - Kooperation zwischen medizinisch-therapeutischem und } \\
\text { betrieblichem System beschränkt sich auf den Zugang } \\
\text { - Informationsverluste im weiteren RTW-Verlauf } \\
\text { - Einseitiger Fokus auf individueller (An-)Passung } \\
\text { - Individualisierung der Erkrankung, ihrer Ursachen und } \\
\text { der erfolgreichen Bewältigung } \\
\text { - } \text { Auslagerung des ,Problems` und seiner Behandlung aus } \\
\text { dem Betrieb } \\
\text { - Beiderseitige (An-)Passung wird in Verantwortung des } \\
\text { Betroffenen gelegt; dieser ist dabei abhängig von der } \\
\text { - Responsivität des Arbeitgebers } \\
\text { - Risiko der Überforderung des Betroffenen } \\
\text { - Mangerfolg ggf. als persönliches Versagen erlebt }\end{array}$ & $\begin{array}{l}\text { - Risiko, dass durch den externen Dritten den Betroffenen die Eigenverant- } \\
\text { wortung genommen wird und Arbeitgeber ihre Verantwortung abgeben } \\
\text { (Gefahr der Externalisierung) } \\
\text { - Aufbau von Parallelstrukturen (externer Dritter als zusätzlicher Akteur zu } \\
\text { medizinisch-therapeutischen und betrieblichen Akteuren); Risiko zur } \\
\text { Verantwortungsdiffusion } \\
\text { - Begrenzte Handlungsmacht des externen Dritten (abhängig von } \\
\text { Akzeptanz und Mitwirken der Arbeitgeber); ggf. auch begrenztes } \\
\text { Handlungswissen (hinsichtl. betrieblicher Strukturen, Prozesse, Kulturen) } \\
\text { - i.d.R. kein expliziter Arbeitsbezug der Regelversorgung } \\
\text { - Ggf. nur bei komplexen Bedarfslagen effizient }\end{array}$ \\
\hline
\end{tabular}

Die größte Stärke des individuumsbezogenen Ansatzes liegt in der Stärkung von Selbstsorge und Selbstmitgefühl, durch die die Betroffenen wieder Selbstwertgefühl, Selbstwirksamkeit und IchStärke zurückgewinnen können. Überwinden die Betroffenen ihre Krise und kehren erfolgreich zurück ins Arbeitsleben, so erleben sie dies als Ergebnis ihrer eigenen Anstrengungen. Misserfolg kann andererseits als persönliches Versagen erlebt werden, da die Wiedereingliederung als ein Prozess der individuellen (An-)Passung ver- standen wird. So besteht die größte Begrenzung des individuumsbezogenen Ansatzes denn auch in der Gefahr der Individualisierung der Erkrankung, ihrer Ursachen und erfolgreichen Bewältigung. Diese Gefahr besteht insbesondere dann, wenn kein funktionierendes BEM und/oder engagierte betriebliche Schlüsselakteure (Betriebsärzte, Schwerbehindertenvertretungen, Führungskräfte o.a.) vorhanden sind. In solchen Fällen kann es zu einer Auslagerung des ,Problems‘ und seiner Behandlung kommen: Betroffene werden 
möglichst schnell identifiziert und an die kooperierenden medizinisch-therapeutischen Einrichtungen verwiesen, in denen sie durch rein individuelle (An-)Passung wieder ,fit' für ihre - unveränderte - Arbeit gemacht werden. Streben Betroffene in solchen Fällen eine Veränderung arbeitsbezogener Belastungen an, so sind sie nicht nur auf sich selbst gestellt, sondern auch komplett abhängig von der Responsivität, Handlungs- und Veränderungsbereitschaft ihrer Vorgesetzten und Arbeitgeber. In Kombination mit einem funktionierenden BEM und/oder dem Engagement betrieblicher Schlüsselakteure, welche die betroffenen Beschäftigten begleiten und im Bedarfsfall mit arbeitsbezogenen Maßnahmen unterstützen, könnte ein auf das Selbstmanagement zielender Behandlungsansatz eine wirksame Unterstützung bei der Rückkehr an den Arbeitsplatz bieten.

Die größte Stärke des systemischen Ansatzes liegt in der gezielt auf den jeweiligen Einzelfall zugeschnittenen Kombination individuums- und arbeitsbezogener Maßnahmen und dem expliziten Bestreben um individuelle wie betriebliche (An-)Passung. Sowohl dem Betroffenem als auch dem Betrieb wird zu diesem Zwecke eine unterstützende Person zur Seite gestellt, die den gesamten Return to Work-Prozess mit plant, gestaltet und koordiniert (Identifikation von Problem-/Bedarfslagen, Aufzeigen von Risikofaktoren, Initiieren geeigneter Angebote der medizinisch-therapeutischen Regelversorgung sowie von betrieblichen Maßnahmen, Moderation/Mediation bei Konflikten am Arbeitsplatz, Vertreten von Interessen Betroffener usw.). Dies kann nicht nur zur Nachhaltigkeit im individuellen Fall beitragen, sondern bietet auch eine mögliche Schnittstelle zur betrieblichen Prävention. Da im systemischen Ansatz bei Bedarf auf Angebote der Regelversorgung psychisch erkrankter Personen zurückgegriffen wird, kann hier jedoch nicht immer gewährleistet werden, dass die in Anspruch genommenen individuumsbezogenen Maßnahmen (wie z. B. Psychotherapie) einen expliziten Arbeits(platz-)bezug aufweisen. Eine weitere Begrenzung des vorgefundenen systemischen Ansatzes liegt in der Abhängigkeit der externen Dritten von der Unterstützungsbereitschaft der betrieblichen Akteure, die die erforderlichen betrieblichen (An-) Passungen immer mittragen bzw. mit umsetzen müssen. Auch für den systemischen Ansatz gilt daher, dass er v. a. dann eine wirksame und nachhaltige Unterstützung bei der Rückkehr an den Arbeitsplatz bieten kann, wenn er auf ein funktionierendes BEM und/ oder engagierte betriebliche Schlüsselakteure trifft und es gelingt, eine gute Balance zwischen individueller Selbst- und betrieblicher Fürsorge herzustellen ( $\triangleright$ Tab. 6).

\section{Implikationen für die Praxis}

Die betriebliche Wiedereingliederung von Beschäftigten mit psychischen Krisen wird wie auch ihre Erkrankung selbst zumeist durch personen- und arbeitsbezogene Faktoren geprägt. Künftig sollten daher solche (Be-)Handlungsansätze verfolgt werden, die neben den individuellen Einstellungen, Haltungen und Verhaltensweisen der Betroffenen auch verstärkt deren Arbeitsbedingungen und -beziehungen in den Fokus nehmen und über das gezielte Zusammenwirken von medizinisch-therapeutischen und betrieblichen Akteuren und Maßnahmen sowohl auf die individuelle als auch betriebliche (An-)Passung abzielen (vgl. [12, 21]). Ziel sollten nicht nur gesunde Beschäftigte, sondern immer auch gesunde Unternehmen sein.
Wie die vorliegende Studie zeigt, stoßen - trotz ihrer Vorteile gegenüber der Regelversorgung - auch stärker vernetzte und arbeitsplatzbezogene Angebote hier noch an ihre Grenzen. Für ihre Weiterentwicklung und künftige Ausweitung auf die Regelversorgung bedarf es v. a.:

1. einer weiteren Information und Sensibilisierung insbesondere relevanter Schlüsselakteure (d.h. betrieblicher und medizinischtherapeutischer Akteure, Leistungsträger, Arbeitnehmer/Betroffene) zum Abbau der Tabuisierung, Stigmatisierung und Diskriminierung psychisch erkrankter Menschen (einschließlich der kritischen Reflexion einseitiger, stereotypisierender Betroffenenbilder),

2. einer konsequenten Berücksichtigung der Themen Arbeit und Wiedereingliederung in der medizinisch-therapeutischen Versorgung psychisch erkrankter Menschen, etwa in der Aus-, Fortund Weiterbildung von Haus-, Fach- und Betriebs-/Werksärzten sowie Therapeuten, in Leitlinien, Therapiestandards, Curricula und Manualen sowie in der Anerkennung und Finanzierung entsprechender Angebote durch Leistungsträger,

3. eines gezielten Wissenstransfers zwischen medizinisch-therapeutischen und betrieblichen Akteuren, um so noch mehr arbeitsmedizinisches Wissen bzw. Arbeitsschutzwissen in die therapeutische Arbeit und andersherum therapeutisch relevantes Wissen in die Ausgestaltung der betrieblichen Rückkehr einfließen lassen zu können,

4. der Förderung betrieblicher Unterstützungsangebote sowie einer stärkeren Einforderung gesetzlicher Schutz- und Fürsorgepflichten mittels geeigneter Informations- und Beratungsstrukturen (zentraler, trägerübergreifender Ansprechpartner für Unternehmen), Anreizsysteme (Zuschüsse, Prämien oder Beitragsnachlässe für besonders engagierte Betriebe, Verlängerung der Lohnfortzahlung) sowie Kontroll- und Sanktionierungsverfahren,

5. des weiteren Ausbaus des Betrieblichen Eingliederungsmanagements sowie der Stärkung und Aufwertung der Rolle von Betriebsärzten im Prozess der betrieblichen Wiedereingliederung,

6. mehr direkter Vernetzung medizinisch-therapeutischer und betrieblicher Akteure im konkreten Einzelfall, einschließlich der konzertierten Planung und Steuerung der Wiedereingliederung und bedarfsorientierten Verschränkung betrieblicher und medizinisch-therapeutischer, arbeitsplatz- und individuumsbezogener, Maßnahmen,

7. der (trägerübergreifend und ggf. arbeitgeberseitig mitfinanzierten) Möglichkeit für alle Beteiligten, bei Bedarf einen externen Dritten als Case Manager/RTW-Koordinator einzuschalten/ zu beauftragen.

\section{Limitationen der Studie}

Die Studie untersucht erstmalig verschiedene Versorgungsnetzwerke und -angebote für Beschäftigte mit psychischen Krisen, beschreibt die Vernetzungsformen und (Be-)Handlungsansätze und deren Stärken und Grenzen im Hinblick auf die Wiedereingliederung der Betroffenen. Mit dem gewählten Studiendesign und methodischem Vorgehen konnten hierbei sowohl die expliziten als 
auch die impliziten Erfahrungs- und Wissensbestände der in die Netzwerke und Angebote involvierten betrieblichen und überbetrieblichen Akteure sowie der Betroffenen berücksichtigt und dadurch umfassendere und tiefergehende Erkenntnisse zu den Angeboten gewonnen werden.

Die Studie weist jedoch auch Limitationen auf, vor deren Hintergrund die Ergebnisse und Schlussfolgerungen zu bewerten sind. So konnte nur eine begrenzte Auswahl an (veröffentlichten) Angeboten untersucht werden, wodurch andere Angebote mit ggf. weiteren Vernetzungsformen und (Be-)Handlungsansätzen mit ihren jeweiligen Stärken und Grenzen unbeachtet bleiben. Da keine kleinen Unternehmen für die Studienteilnahme gewonnen werden konnten (weil sie in den fokussierten Versorgungsnetzwerken und -angeboten unterrepräsentiert sind, aber auch weil ggf. intensivere Rekrutierungsbemühungen von Nöten gewesen wären), bleibt zudem unklar, welche Besonderheiten sich hier in Vernetzung und Behandlung ergeben und wie/unter welchen Bedingungen kleine Unternehmen und ihre Beschäftigten von stärker vernetzten und arbeitsplatzbezogenen Angeboten profitieren können. Zum realisierten Sample ist anzumerken, dass 1. bei einem Angebot (Angebot 4) keine Gruppendiskussion mit betrieblichen Akteuren durchgeführt werden konnte, 2. zwei der Gruppendiskussionen auf Betriebs- und Einrichtungsseite (Angebote 1 und 5) vergleichsweise gering und im Hinblick auf mögliche Akteure und Professionen recht homogen besetzt waren, und 3. auf Seite der Betroffenen fast ausschließlich Menschen mit Depressionen bzw. depressiver Symptomatik in die Studie eingeschlossen wurden. Mit Blick auf die weitere Dissemination, die v. a. von finanziellen und rechtlichen Rahmenbedingungen abhängt, hätte die Studie schließlich vom Einbezug einer weiteren Akteursgruppe, die der (potentiellen) Leistungsträger und politischen Entscheidungsträger, profitieren können.

\section{KERNBOTSCHAFT}

Die Entstehung psychischer Erkrankungen ist wie auch die betriebliche Wiedereingliederung zumeist ein multifaktorielles Geschehen. Die Komplexität von Erkrankung und Rückkehr müssen daher anerkannt und individuelle wie betriebliche (An-)Passung gleichermaßen berücksichtigt werden. Dazu sollte die Zusammenarbeit zwischen behandelnden Ärzten, Therapeuten und Betriebsärzten sowie weiteren zentralen Schlüsselakteuren im BEM weiter ausgebaut und verbessert werden. Die Einbindung externer Dritter, die eine professionelle Begleitung im RTW-Prozess ermöglichen, erscheint hierbei zielführend.

\section{Finanzielle Unterstützung}

Die Studie wurde von der Hans-Böckler-Stiftung im Rahmen des Forschungsverbundes „Neue Allianzen zum Erhalt der Beschäftigungsfähigkeit“ gefördert (Projekt-Nr.: 2016-980-4).
Interessenkonflikt

Die AutorInnen geben an, dass kein Interessenkonflikt besteht.

Literatur

[1] Jacobi F, Höfler M, Strehle J et al. Psychische Störungen in der Allgemeinbevölkerung. Studie zur Gesundheit Erwachsener in Deutschland und ihr Zusatzmodul Psychische Gesundheit (DEGS1MH). Nervenarzt 2014; 85: 77-87. doi: 10.1007/s00115-013-3961-y

[2] Wege N, Angerer P. Psychische Erkrankungen - Auswirkungen auf die Arbeitsfähigkeit und Versorgung psychisch erkrankter Erwerbstätiger. Psychiatrie 2013; 10: 71-81. doi: 10.1055/s-0038-1670858

[3] Rothe I, Adolph L, Beermann B et al. Psychische Gesundheit in der Arbeitswelt - Wissenschaftliche Standortbestimmung. Dortmund: Bundesanstalt für Arbeitsschutz und Arbeitsmedizin; 2017

[4] Semmer NK, Udris I. Bedeutung und Wirkung von Arbeit. In: Schuler H, Hrsg. Lehrbuch Organisationspsychologie. Bern: Huber; 2004

[5] Bundesministerium für Arbeit und Soziales (BMAS), Bundesanstalt für Arbeitsschutz und Arbeitsmedizin (BAuA), Hrsg. Sicherheit und Gesundheit bei der Arbeit 2014. Unfallverhütungsbericht Arbeit. Dortmund, Berlin, Dresden: BAuA; 2016

[6] Wege N, Janssen B, Rothermund E. Betriebsnahe Versorgungsnetzwerke: Beispiele guter Praxis in Deutschland. In: Angerer P, Glaser J, Gündel $\mathrm{H}$ et al, Hrsg. Psychische und psychosomatische Gesundheit in der Arbeit. Wissenschaft, Erfahrungen und Lösungen aus Arbeitsmedizin, Arbeitspsychologie und Psychosomatischer Medizin. Heidelberg: Ecomed MEDIZIN; 2014

[7] Arends I, Bruinvels DJ, Rebergen DS et al. Interventions to facilitate return to work in adults with adjustment disorders. Cochrane Database Syst Rev 2012. doi: 10.1002/14651858.CD006389.pub2

[8] Nieuwenhuijsen K, Faber B, Verbeek JH. Interventions to improve return to work in depressed people. Cochrane Database Syst Rev 2014; CD006237. doi: 10.1002/14651858.CD006237.pub3

[9] van Vilsteren M, van Oostrom SH, de Vet HC et al. Workplace interventions to prevent work disability in workers on sick leave. Cochrane Database Syst Rev 2015. doi: 10.1002/14651858. CD006955.pub3

[10] Joyce M, Modini M, Christensen $\mathrm{H}$ et al. Workplace interventions for common mental disorders: a systematic meta-review. Psychol Med 2016; 46: 683-697. doi: 10.1017/S0033291715002408

[11] Cullen KL, Irvin E, Collie A et al. Effectiveness of Workplace Interventions in Return-to-Work for Musculoskeletal, Pain-Related and Mental Health Conditions: An Update of the Evidence and Messages for Practitioners. J Occup Rehabil 2018; 28: 1-15. doi: 10.1007/ s10926-016-9690-x

[12] Stegmann R, Schröder U.B. Psychischer Erkrankungen in der Arbeitswelt: Wiedereingliederung nach einer psychischen Krise. Ergebnisse einer qualitativen Studie. Arbeitsmedizin, Sozialmedizin, Umweltmedizin ASU - Zeitschrift für medizinische Prävention 2016; 51: 660-668

[13] Stock Gissendanner S, Weiß C, Herten B, Wrage W. et al. Eine psychosomatische Sprechstunde für die regionale betriebsnahe Versorgung: Evaluation und Empfehlungen. Eingereicht August 2019: Arbeitsmedizin, Sozialmedizin, Umweltmedizin ASU - Zeitschrift für medizinische Prävention

[14] Schwarz B, Wegewitz U. Rückkehr zur Arbeit nach psychischer Krise. Betriebsnahe Versorgungsnetzwerke unter der Lupe. Arbeitsmedizin, Sozialmedizin, Umweltmedizin ASU - Zeitschrift für medizinische Prävention 2018; 53: 368-371 
[15] Bohnsack R. Generation, Milieu und Geschlecht. Ergebnisse aus Gruppendiskussionen mit Jugendlichen. Opladen: Leske+Budrich; 1989

[16] Bohnsack R. Rekonstruktive Sozialforschung. Einführung in qualitative Methoden. Opladen \& Farmington Hills: Verlag Barbara Budrich; 2010

[17] Bohnsack R, Nentwig-Gesemann I, Nohl AM. Die dokumentarische Methode und ihre Forschungspraxis. Wiesbaden: Springer; 2013

[18] Przyborski A, Wohlrab-Sahr M. Qualitative Sozialforschung: ein Arbeitsbuch. München: Oldenburg; 2014

[19] Kuckartz U. Qualitative Inhaltsanalyse. Methoden, Praxis, Computerunterstützung. Weinheim, Basel: Beltz Juventa; 2014
[20] Schreier M. Varianten qualitativer Inhaltsanalyse: Ein Wegweiser im Dickicht der Begrifflichkeiten. Forum Qualitative Sozialforschung/ Forum: Qualitative Social Research 15(1), Art. 18. http://nbn-resolving.de/urn:nbn:de:0114-fqs1401185 [Datum des Zugriffs: 20.08.2019]

[21] Stegmann R, Schulz I, Schröder UB. Return to Work nach psychischer Erkrankung. Mixed-Methods-Follow-Up-Studie: Determinanten einer erfolgreichen Wiedereingliederung, Ergebnisse der qualitativen Teilstudie. 28. Rehabilitationswissenschaftliches Kolloquium, DRV-Schriften, Band 117 